\title{
The role of genetic mutations on genes PAX2 \& PAX6 in cyclopia syndromee
}

\author{
Shahin Asadi ${ }^{1,2 *}$ \\ ${ }^{1}$ Medical Genetics-Harvard University, Director of the Division of Medical Genetics and Molecular Optogenetic Research, USA \\ ${ }^{2}$ Division of Medical Genetics and Molecular Pathology Research, Harvard University, Boston Children's Hospital, USA
}

\begin{abstract}
Cyclopia syndrome typically coexists with holoprosencephaly. This ocular lesion may present as complete ocular fusion in a single orbit or as two eyes in a single orbit. Eye defects linked with cyclopia syndrome include colobomas (gaps) in the iris, retina, and optic nerve; inconstant optic nerve numbers (either one or two is possible), and an absent or abnormal optic chiasm. Cyclopia syndrome is caused by mutations in the PAX2 and PAX6 genes. Cyclopia syndrome occurs when the rostral (anterior) portion of the notochord and adjacent mesoderm are deficient in mass. This shortage leads to the aberrant induction of the forebrain tissues followed by severe derangement of midline facial development.
\end{abstract}

\section{Overview of cyclopia syndrome}

Cyclopia syndrome is a rare disorder of holoprosencephaly (fracture of the brain in the fetus) that occurs with birth defects. In this disease, the orbit of the eye is divided into two very close caverns, with either the monocular organism being seen or the binoculars being closely related. The syndrome affects both animals and humans, with the frequency in animals 1 in every 16,000 births and in the human fetus 1 in 250 births (Figure 1) [1].

\section{Signs and symptoms of cyclopia syndrome}

Typically, patients with cyclopia syndrome have a long nose or inadequate nose in the form of a long snout. Patients with cyclopia syndrome have one eye in the middle of the forehead or two eyes completely clinging. The ears are bent or fractured or crumpled inward, and most patients have a fleshy appendage 3 to $5 \mathrm{~cm}$ in the middle of their forehead [1].

Cyclopia syndrome most commonly involves embryos that are either aborted or born at the same age as the fetus and, in good fortune, will be certain shortly after their death. Cyclopia syndrome is a rare genetic disorder that has been reported in only a few cases in

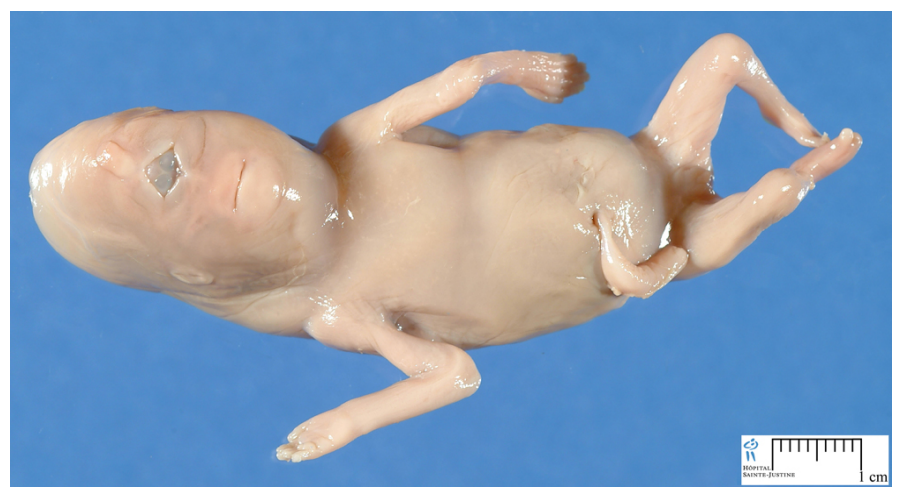

Figure 1. Picture of the fetus with Cyclopia syndrome the medical world, and most are preserved at the vrolik Amsterdam Museum of Medicine in the Netherlands (Figure 2) [1].

Animals that develop cyclopia syndrome include farm animals such as horses, sheep, pigs, and chickens. In these animals, like humans, the nasal organs form inefficiently, which shortly after birth causes the airway to pass through the respiratory tract, resulting in suffocation $[1,2]$.

\section{Etiology of cyclopia syndrome}

One of the major causes of cyclopia syndrome can be increased maternal age during pregnancy and the susceptibility of cytopenia to cyclopia syndrome in post-fertilization and zygote cell formation, as well as the use of toxic or sedative alkaloids. Maternal pregnancy can increase the susceptibility of the fetus to cyclopia syndrome. In fact, most people with cyclopia syndrome are due to the mother's mistake during pregnancy in using herbs such as veratrum californicum to improve vomiting and cramping and poor blood circulation. In other words, this plant has alkaloids, although it is known as a natural painkiller in general. Of course, the use of this medicinal plant during pregnancy has no adverse effect on women and men, but it was very dangerous during pregnancy due to its alkaloid properties and is a potent teratogenic agent. This alkaloid property of the plant disturbs the mitotic and spindle filament cycles, resulting in the failure of the eye,

${ }^{\star}$ Correspondence to: Shahin Asadi, Medical Genetics-Harvard University. Director of the Division of Medical Genetics and Molecular Optogenetic Research, USA; Division of Medical Genetics and Molecular Pathology Research, Harvard University, Boston Children's Hospital, USA, E-mail: shahin.asadi1985@gmail.com

Key words: cyclopia Syndrome, genetic Mutations, PAX2, PAX6 Genes, holoprosencephaly

Received: February 24, 2020; Accepted: March 02, 2020; Published: March 05, 2020 
nose, and ear cavity to form successfully, as well as the correct shape of the brain (Figure 3) $[1,3]$.

\section{Molecular genetics of cyclopia syndrome}

SHH gene, as the regulator of expression and synthesis of proteins of the nervous system for the formation of the brain and PAX6 gene, is the correct state of synthesis of the eyes which, due to chromosomal abnormalities, are mutated and lose their normal function. The SHH gene is located on the long arm of chromosome 7 at $7 \mathrm{q} 36.3$, and in addition to the above, the gene regulates the correct shape of the hands and nose. The SHH gene plays a key role in initiating the expression of other genes such as PAX6 and PAX2 in the cell (Figure 4 and 5) [1,4].

The PAX6 gene acts as a natural protein for the eyes. The gene is located on the short arm of chromosome 11 in $11 \mathrm{p} 13$. This gene plays a key role in the evolution of the eyes to the natural form, both in humans and animals (Figure 6-8) [1,5].

Any abnormality in this gene, including nucleotide deletion or deletion or insertion, will lead to impaired vision or blindness. Patients with cyclopia syndrome, on the short arm of chromosome 11 in the $11 \mathrm{p} 11$ and $11 \mathrm{p} 13$ regions, have mutations that occur and cause visual and visual developmental disorders $[1,6]$.

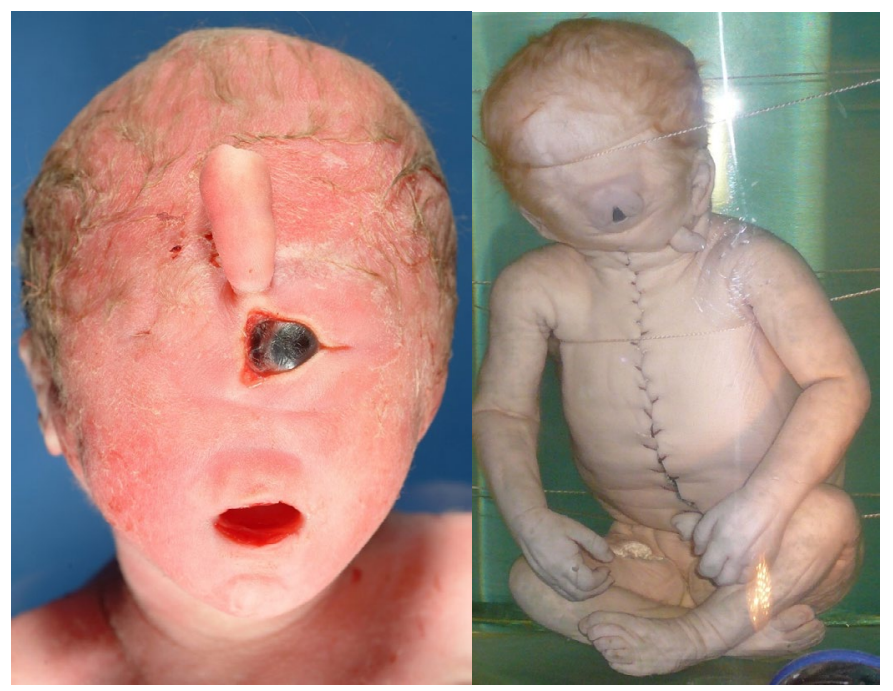

Figure 2. Image of human with cyclopia syndrome with indicative features

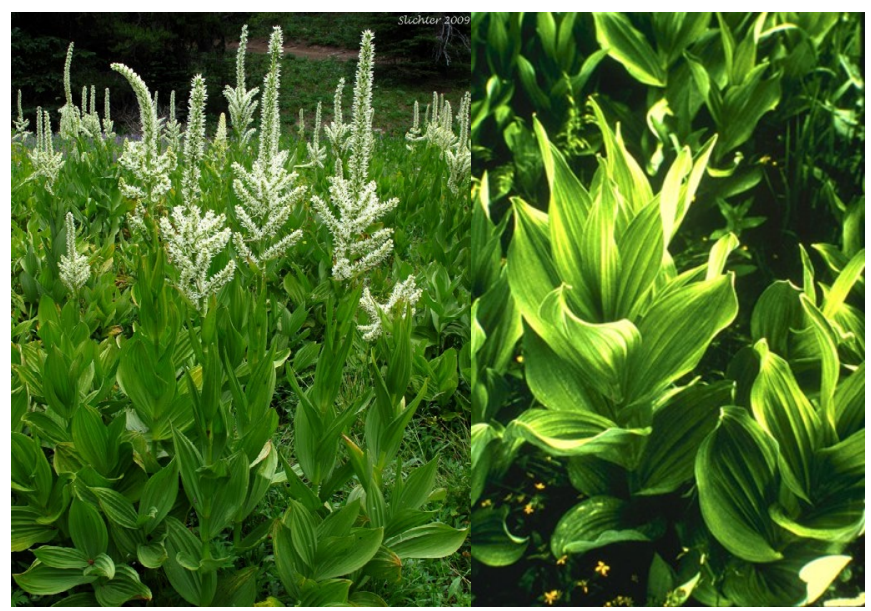

Figure 3. Picture of Veratrum medicinal plant

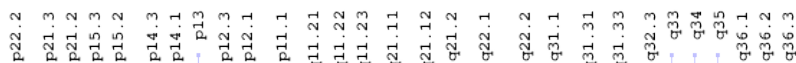

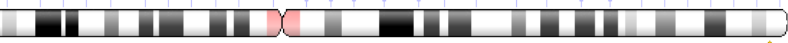

Figure 4. Schematic overview of chromosome 7 where the SHH gene is located in the long arm of chromosome $7 \mathrm{q} 36.3$

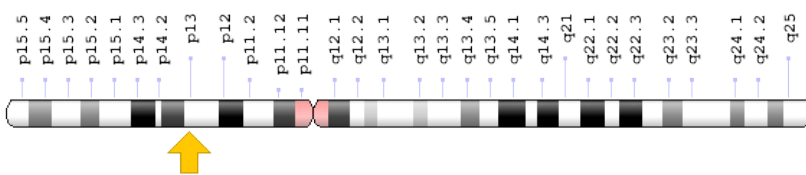

Figure 5. Schematic overview of chromosome 11 where the PAX6 gene is located in the short arm of chromosome $11 \mathrm{p} 13$

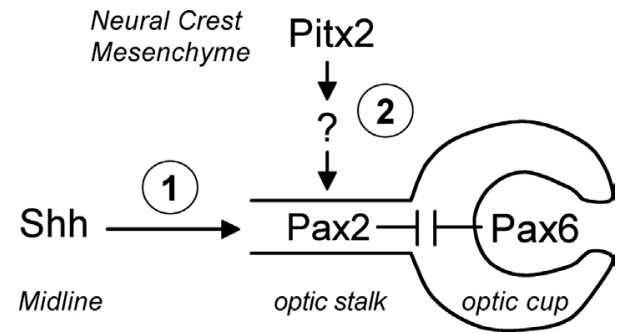

Figure 6. Schematic overview of the SHH gene pathway for induction of PAX2 and PAX6 gene expression in eye tissue development
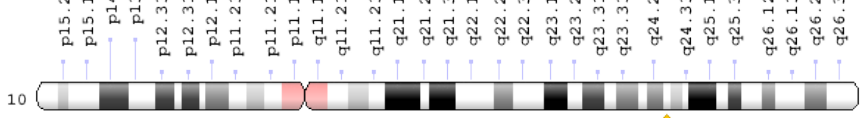

Figure 7. Schematic view of chromosome 10 where the PAX2 gene is located on the long arm of chromosome 10q24.31

(a)

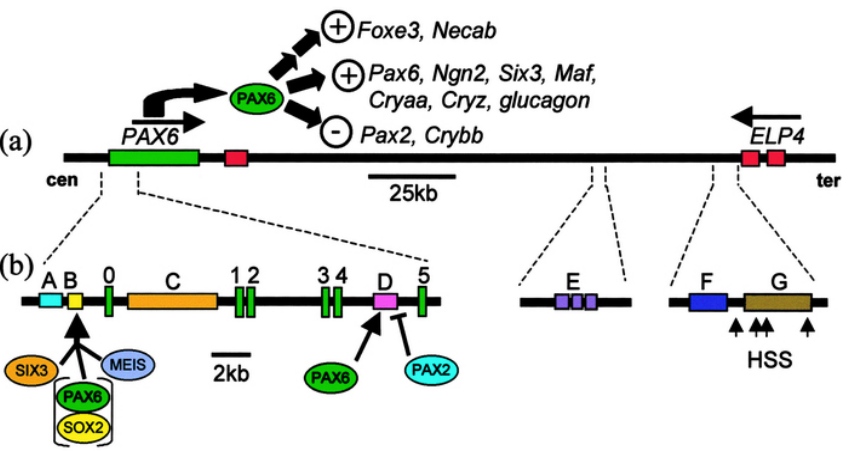

Figure 8. Schematic of the molecular mechanism of function of the PAX6 gene

\section{Diagnosis of cyclopia syndrome}

Cyclopia syndrome is diagnosed based on facial features findings with imaging techniques such as ultrasound. Prenatal diagnosis is also possible by molecular genetic testing of amniocentesis fluid and fetal placenta chorionic villi $[1,7]$.

\section{History of cyclopia syndrome}

The word cyclops in ancient Greek means an eye circle referring to an athlete in ancient Greek mythology. This hero had one eye in the center of the forehead. The name of this hero was Polyphemus. He was 
the son of Uranus and Gaia of the great Titans of ancient Greece (Figure 9) $[1,8]$.

But the first report of cyclopia syndrome goes back to 1665 on a horse in the UK. Then in March 1793, a 46 - year - old woman in Sweden gave birth to a child with cyclopia syndrome who died after two hours. On December 28, 2005, a kitten in Redmond, USA, was born with cyclopia syndrome, who died a day after giving birth. In 2006, a baby girl in India was born with cyclopia syndrome who died a day after giving birth. In 2011, an embryo with cyclopia syndrome was discovered from the belly of a giant hunting shark in Mexico. In 2012, a kitten was born with cyclopedia syndrome who died after two hours. Cyclopia syndrome has symptoms similar to patau syndrome. But the phenotype of cyclopia Syndrome is much more severe than that of patau syndrome and the half-life of cyclopia syndrome patients is shorter than that of patau syndrome. The major genes involved in cyclopia syndrome are: SHH, ZIC2, SLX3, and TGIF1 (Figure 10) [1,9].

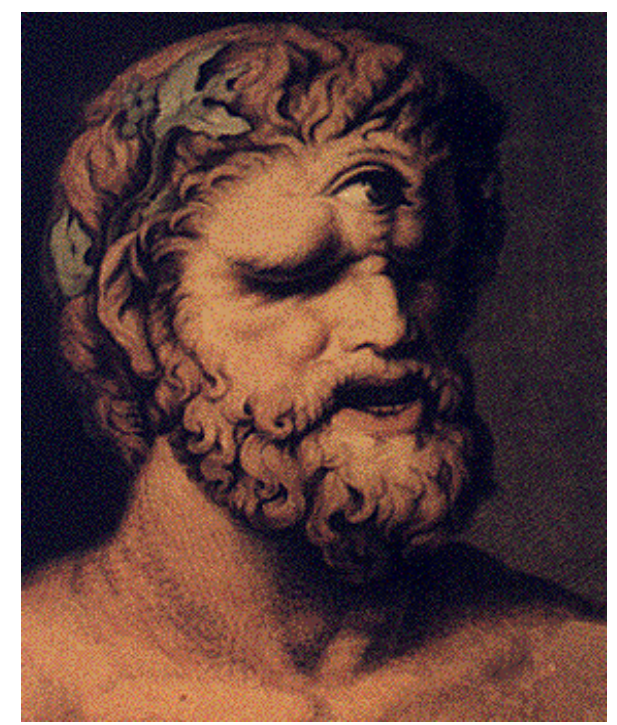

Figure 9. Painting image of the Athenian god Polyphemus, who had an eye in the middle of the forehead

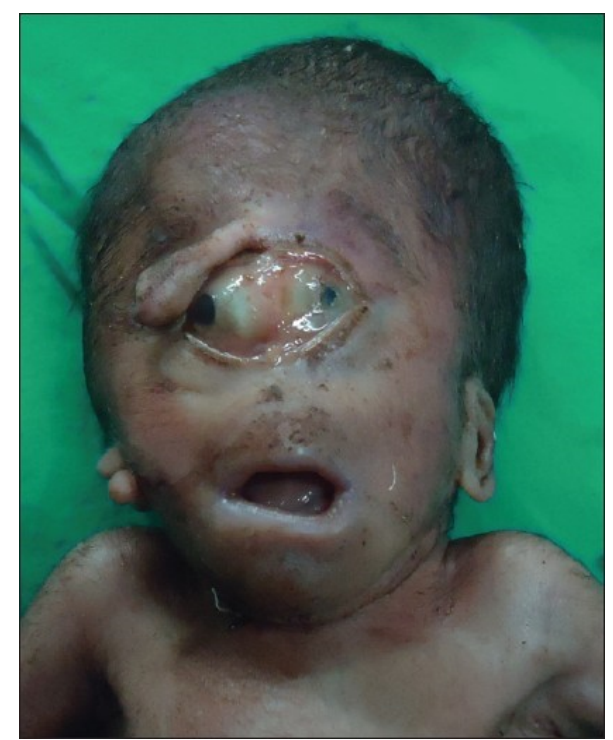

Figure 10. Image of a baby with cyclopia syndrome

\section{Discussion and conclusion}

Cyclopia (also cyclocephaly or synophthalmia) is a rare form of holoprosencephaly and is a congenital disorder (birth defect) characterised by the failure of the embryonic prosencephalon to properly divide the orbits of the eye into two cavities. It is the severest facial expression of the holoprosencephaly syndrome. Its incidence is 1 in 100000 in newborns. Typically, the nose is either missing or replaced with a non-functioning nose in the form of a proboscis. Such a proboscis generally appears above the central eye, or on the back, and is characteristic of a form of cyclopia called rhinencephaly or rhinocephaly. Most such embryos are either naturally aborted or are stillborn on delivery. Some cases of cyclopia have been associated with a rare chromosomal condition called 'Patau Syndrome' (trisomy 13). SHH (Sonic Hedgehog Gene Regulator) is involved in the separation of the single eye field into two bilateral fields. Although not proven, it is thought that $\mathrm{SHH}$ emitted from the prechordal plate suppresses Pax6 which causes the eye field to divide into two. If the SHH gene is mutated, the result is cyclopia, a single eye in the centre of the face [1-10].

\section{References}

1. Asadi S, Jamali M, Bagheri R, Sadeh Dell S, Tohidirad M (2017) Book of Pathology in Medical Genetics Vol 1 (A-L), Amidi Publications.

2. Observables upon a Monstrous Head (1665) Philosophical Transactions of the Royal Society (1665-1678) 1: 85-56.

3. Marigo V, Roberts DJ, Lee SM, Tsukurov O, Levi T, et al. (1995) "Cloning, expression, and chromosomal location of SHH and IHH: two human homologues of the Drosophila segment polarity gene hedgehog". Genomics $28: 44-51$.

4. Nüsslein VC, Wieschaus E (1980) "Mutations affecting segment number and polarity in Drosophila". Nature 287: 795-801.

5. Krauss S, Concordet JP, Ingham PW (1993) "A functionally conserved homolog of the Drosophila segment polarity gene hh is expressed in tissues with polarizing activity in zebrafish embryos". Cell 75: 1431-1444.

6. Echelard Y, Epstein DJ, St-Jacques B, Shen L, Mohler J, et al. (1993). "Sonic hedgehog, a member of a family of putative signaling molecules, is implicated in the regulation of CNS polarity". Cell 75: 1417-1430.

7. Herzog W, Zeng X, Lele Z, Sonntag C, Ting JW, et al. (2003) "Adenohypophysis formation in the zebrafish and its dependence on sonic hedgehog". Dev Biol 254: 36-49.

8. Rash BG, Grove EA (2007) "Patterning the dorsal telencephalon: a role for sonic hedgehog?". The Journal of Neuroscience 27: 11595-11603.

9. Scholpp S, Wolf O, Brand M, Lumsden A (2006) "Hedgehog signalling from the zona limitans intrathalamica orchestrates patterning of the zebrafish diencephalon". Development 133: 855-864

10. McMahon JA, Takada S, Zimmerman LB, Fan CM, Harland RM, et al. (1998) Nogginmediated antagonism of BMP signaling is required for growth and patterning of the neural tube and somite. Genes \& development 12: 1438-1452.

Copyright: (C2020 Asadi S. This is an open-access article distributed under the terms of the Creative Commons Attribution License, which permits unrestricted use, distribution, and reproduction in any medium, provided the original author and source are credited. 